\title{
Stage IB Carcinoma of the Cervix with Lymph Node Metastases
}

\author{
Keith Y. Terada, M.D., George W. Morley, M.D. \\ And James A. Roberts, M.D. \\ Department of Obstetrics and Gynecology, University of Michigan Hospitals, 1500 East Medical \\ Center Drive, Ann Arbor, Michigan 48109-0718
}

Received March 10. 1987

\begin{abstract}
From January 1, 1946, through January 1, 1986, at the University of Michigan, 60 patients were treated with Stage IB (FIGO) carcinoma of the cervix with lymph node metastases. Clinical variables were examined with regard to long-term survival. Prognostically significant variables were (1) highest level of lymph node involvement and (2) tumor histology. Patients with common iliac or periaortic node involvement had an actuarial survival at 5 years of $14 \%$; 5-year survival for patients with only pelvic node involvement (external iliac, hypogastric, or obturator) was $68 \%$. Patients with adenocarcinoma or adenosquamous histology had an actuarial survival at 5 years of $16 \% ; 5$-year survival for patient with squamous cell carcinoma was $71 \%$. Overall survival for all patients with lymph node involvement was $61 \%$. For patients undergoing radical hysterectomy as primary treatment, postoperative adjuvant radiotherapy did not significantly improve survival. (c) 1988 Academic Press, Inc.
\end{abstract}

\section{INTRODUCTION}

Regional lymph node involvement has a significant impact upon survival in Stage IB (FIGO) carcinoma of the cervix. Overall 5-year survival for Stage IB carcinoma of the cervix is presently 80 to $90 \%$ [1]. When lymph node metastases are present, however, long-term survival falls to less than $60 \%$ [2]. The present study was undertaken to review the clinical factors affecting survival among those patients with Stage IB carcinoma of the cervix with lymph node metastases.

\section{MATERIALS AND METHODS}

During the period January 1, 1946, through January 1, 1986, 362 patients underwent radical hysterectomy and pelvic lymphadenectomy at the University of Michigan Medical Center for Stage IB carcinoma of the cervix. Forty-seven patients $(13 \%)$ were found to have lymph node metastases. Eleven patients with Stage IB cervical carcinoma had lymph node metastases documented at the time of exploratory laparotomy. These patients were found to have fixed or confluent nodes judged to be unresectable; biopsies were done to confirm metastatic tumor. One patient had nodal metastases noted on pretreatment lymphangiogram and confirmed by fine-needle aspiration. One patient underwent an extrafascial hysterectomy and selective lymph node biopsies for a preoperative diagnosis of 
TABLE 1

Tumor Histology in Relation to Highest Level of Lymph Node Involvement

\begin{tabular}{lccr}
\hline & $\begin{array}{c}\text { Squamous cell } \\
\text { carcinoma }\end{array}$ & Adenocarcinoma & Total \\
\hline Pelvic (obturator, & & & \\
$\quad$ hypogastric, external iliac) & 43 & 0 & 53 \\
Common iliac & 2 & $\frac{3}{13}$ & $\frac{5}{60}$ \\
Periaortic & $\frac{2}{47}$ & 10 & $\frac{5}{27 a l}$ \\
\hline
\end{tabular}

${ }^{a}$ Including adenosquamous.

adenocarcinoma of the endometrium; the surgical specimen revealed a primary endocervical adenocarcinoma with lymph node metastases. There were, therefore, 60 patients available for review with Stage IB carcinoma of the cervix with lymph node metastases.

The ages of the patients ranged from 20 to 76 with a mean age of 41 . There were 13 patients with adenocarcinoma or adenosquamous carcinoma and 47 patients with squamous cell carcinoma. Two patients (3\%) had extension to the level of the common iliac node group; 5 patients $(8 \%)$ had extension up to the periaortic node group. Tumor histology in relation to highest level of lymph node involvement is indicated in Table 1. The difference in the distributions of the highest level of node involvement when stratified by tumor histology is not statistically significant.

All patients underwent primary treatment with curative intent. Forty-seven patients underwent radical hysterectomy with pelvic lymphadenectomy. Of these 47 patients, 20 received postoperative irradiation. Seven patients were treated primarily with radiotherapy. Four patients were treated with a combination of radiotherapy and chemotherapy. One patient underwent an extrafascial hysterectomy and postoperative radiotherapy. One patient underwent radiotherapy followed by a selective lymph node resection as primary treatment.

The technique of performing the radical hysterectomy has been previously described [1]. Patients undergoing postoperative irradiation received $5000 \mathrm{rad}$ megavoltage teletherapy to a pelvic field. Primary radiotherapy was administered combining intracavitary sources with external beam irradiation.

Clinical charts and tumor board recommendations were reviewed to assess selection factors in determining which patients undergoing radical hysterectomy received postoperative irradiation. The only significant selection factor identified was the time period in which patients received treatment. One of 21 patients (5\%) undergoing treatment prior to 1975 received postoperative irradiation. Nineteen of 26 patients $(76 \%)$ treated after 1975 received postoperative irradiation. This difference was statistically significant ( $P<0.005$ by chi-square analysis).

Five-year survivals were calculated using the actuarial method of Berkson and Gage [3]. Survival distributions were compared using Mantel and Haenszel's chi square test [4]. 


\section{RESULTS}

Overall actuarial survival at 5 years for patients with Stage IB carcinoma of the cervix with lymph node involvement was $61 \%$. When only the pelvic nodes were involved (external iliac, hypogastric, or obturator), 5-year survival was $68 \%$. When there was extension to involve the common iliac or periaortic lymph nodes, 5-year survival was $14 \%$. This difference was statistically significant ( $P$ $<0.005$ ).

Five-year survival in patients with adenocarcinoma or adenosquamous carcinoma was $16 \%$; for patients with squamous cell carcinoma 5 -year survival was $71 \%$. This difference was statistically significant $(P<0.005)$. The difference in survival distributions remained statistically significant even when patients with common iliac or periaortic node involvement were excluded from the analysis.

Histologic grade did not affect survival. Multiplicity of node involvement, i.e., metastatic tumor identified in single versus multiple lymph nodes, did not affect survival. Also, bilaterality of lymph node involvement did not correlate with survival.

For patients undergoing radical hysterectomy with and without postoperative radiotherapy, 5-year survivals were 72 and $64 \%$, respectively. This difference was not statistically significant. Five-year survival for patients undergoing primary radiotherapy was $43 \%$; when compared to patients undergoing radical hysterectomy, this difference was not statistically significant.

Four patients underwent combined treatment with radiotherapy and chemotherapy. One patient received concurrent treatment with hydroxyurea; the other three patients were treated in a sequential manner. The outcome for these patients is summarized in Table 2.

One patient demonstrated metastatic tumor in a right obturator lymph node on pretreatment lymphangiogram; this was confirmed by fine-needle aspiration. She was treated with 5000 rad four-field external beam irradiation, intracavitary cesium, and an additional boost to the right pelvic sidewall. A selective lymph node resection was then performed revealing focal residual carcinoma; ipsilateral external iliac and common iliac nodes were negative. The patient remains free of disease at 19 months.

Sites of recurrence, stratified by tumor histology, are summarized in Table 3. One patient with a pelvic sidewall recurrence survived following radiotherapy; all other patients that developed recurrent tumor died of disease. All recurrences in patients treated with radical hysterectomy alone had pelvic disease as a component of the recurrence. Recurrences after radical hysterectomy with postoperative radiotherapy involved the pelvis less frequently, occurring in only $50 \%$ of patients with recurrent disease.

\section{DISCUSSION}

The incidence of lymph node metastases in patients with Stage IB carcinoma of the cervix is estimated to be approximately 15\% [5]. The present series confirms other reports that 5 year survival in this group of patients approximates $60 \%[2,6]$. In the present series, the two most significant factors affecting survival 


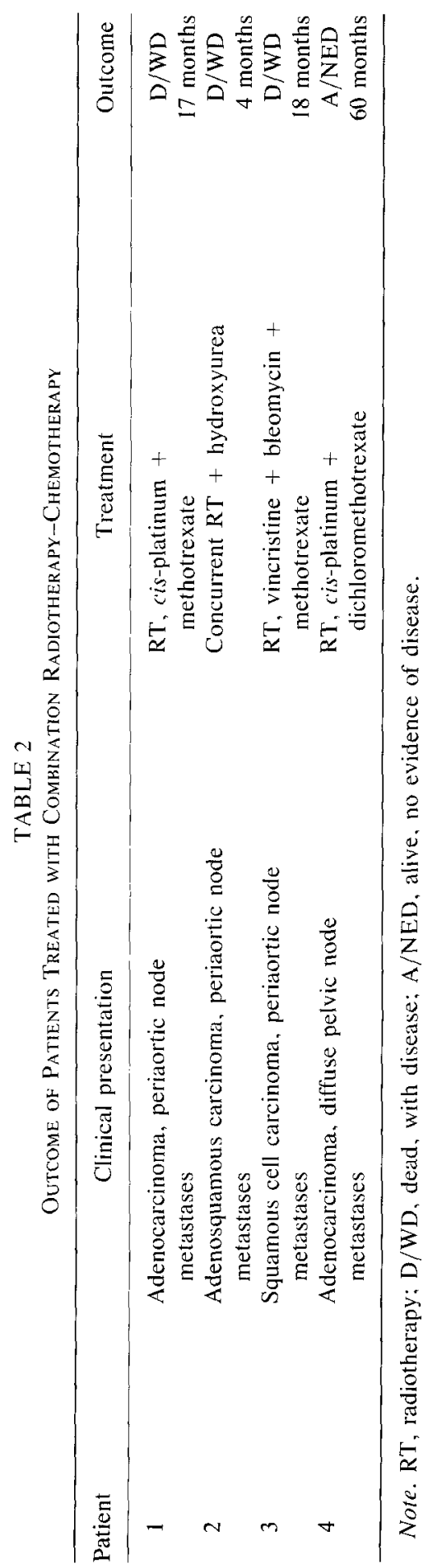


TABLE 3

Sites of RecurRence

\begin{tabular}{lccc}
\hline & $\begin{array}{c}\text { Squamous cell } \\
\text { carcinoma }\end{array}$ & Adenocarcinoma & Total \\
\hline Pelvis & 4 & 1 & 5 \\
Distant & 1 & 4 & 5 \\
Pelvis and distant & 7 & 3 & 10 \\
Not specified & 3 & 0 & 3 \\
\hline
\end{tabular}

"Including adenosquamous.

were (1) the highest level of lymph node involvement and (2) tumor histology. Common iliac or periaortic lymph node involvement indicated a poor long-term survival. Five-year survival in this group of patients was $14 \%$. Others have reported similar survival rates in patients with tumor extension to the common iliac or periaortic level [2,7]. Extension to involve this level of nodes can be an indication of systemic disease and therefore may not be effectively treated with regional therapy. Addition of chemotherapy to the treatment program would seem logical in attempting to improve salvage in this group of patients. In the present series, three of seven patients with nodes involved at this level underwent combined radiotherapy-chemotherapy as primary treatment. All three patients died of recurrent disease; two recurred with distant metastases. The development of more effective chemotherapeutic agents for carcinoma of the cervix may help improve outcome in this group of patients.

In the present series, the prognosis for patients with Stage IB carcinoma of the cervix with lymph node metastases correlated significantly with tumor histology. Patients with adenocarcinoma or adenosquamous tumors had an actuarial survival at 5 years of $16 \%$, significantly worse than for patients with squamous cell carcinoma. Berek et al. have reported a $10 \%$ 5-year survival in patients with adenocarcinoma of the cervix with lymph node metastases [8]. In the present study, seven of eight patients with recurrent adenocarcinoma had distant metastases as a component of recurrence. This confirms Berek's impression that lymph node involvement with adenocarcinoma of the cervix often indicates systemic disease [8]. Logically, consideration should be given to treating these patients with chemotherapy. Clearly, additional clinical trials are needed in this area.

In the present study the addition of postoperative radiotherapy did not improve survival in patients undergoing radical hysterectomy and pelvic lymphadenectomy. Restrospectively, there was no significant difference in the clinical characteristics between the two groups other than the time period of treatment. It appears that recently a greater proportion of patients treated at the University of Michigan received adjunctive radiotherapy for positive lymph nodes; the reason for this trend is unclear. Morrow, reporting on a collective series of 174 patients with Stage IB cervical carcinoma with pelvic node metastases was also unable to demonstrate any significant improvement in survival for patients undergoing postoperative radiotherapy [6]. Martimbeau, although unable to demonstrate an improved survival in such patients, recommends postoperative adjunctive ra- 
diotherapy since it is difficult to completely resect all of the pelvic lymphatics [2]. In the present series, postoperative radiotherapy appeared to improve regional tumor control; this did not correlate with a significant improvement in long-term survival, however.

There were seven patients in the study treated with primary radiotherapy. Although survival in this group was lower ( $43 \%$ at 5 years), the difference was not statistically significant, suggesting that treatment modality was not a primary determinant of survival. Furthermore, the lower survival reflects the selection bias of patients treated with radiotherapy in this series. Three patients had common iliac or periaortic node involvement and four patients had fixed, confluent pelvic node involvement. Clearly, then, radiotherapy can effectively sterilize regional lymphatics, occasionally even when tumor involvement is extensive. This confirms the impression of others $[2,9]$ and indicates a role for radiotherapy in the primary treatment of such individuals.

Of interest in this regard is the single patient who had nodal metastases indentified by pretreatment lymphangiogram. The use of noninvasive imaging techniques when combined with fine-needle aspiration may be useful in identifying patients with regional node involvement $[10,11]$. Treatment can then be individualized based on the documented extent of disease.

In summary, the overall survival for patients with Stage IB carcinoma of the cervix with lymph node metastases is $61 \%$. Patients with tumor extension to the common iliac or periaortic nodes are at particularly high risk for recurrence. Patients with adenocarcinoma or adenosquamous histology, regardless of level of node involvement, are also at high risk for recurrence. Further investigation needs to be carried out to improve salvage in these high risk groups. Radiotherapy can effectively treat patients with regional lymphatic involvement; noninvasive imaging studies may be useful adjuncts in identifying such individuals.

\section{REFERENCES}

1. Morley, G. W., and Seski, J. C. Radical pelvic surgery versus radiation therapy for Stage I carcinoma of the cervix (exclusive of microinvasion), Amer. J. Obstet. Gynecol. 126, 785 (1976).

2. Martimbeau, P. W., Kjorstad, K. E., and Iversen, T. Stage IB carcinoma of the cervix, The Norwegian Radium Hospital. II. Results when pelvic nodes are involved, Obstet. Gynecol. 60, 215 (1982).

3. Berkson, J., and Gage, R. P. Calculation of survival rates for cancer, Proc. Staff Meet Mayo Clinic 25, 270 (1950).

4. Mantel, N., and Haenszel, W. Statistical aspects of the analysis of the data from retrospective studies of disease, $J$. Natl. Cancer Inst. 22, 719 (1959).

5. Plentl, A. A., and Friedman, E. A. Clinical significance of cervical lymphatics, in Lymphatic system of the female genitalia, Saunders, Philadelphia, p. 85-115 (1971).

6. Morrow, C. P. Is pelvic radiation beneficial in the postoperative management of Stage IB squamous cell carcinoma of the cervix with pelvic node metastasis treated by radical hysterectomy and pelvic lymphadenectomy? Gynecol. Oncol. 10, 105, 1980.

7. Ballon, S. C., Berman, M. C., Lagasse, L. D., Petrilli, E. S., and Castaldo, T. W. Survival after extraperitoneal pelvic and periaortic lymphadenoectomy and radiation therapy in cervical carcinoma, Obstet. Gynecol. 57, 90 (1981).

8. Berek, J. S., Hacker, N. F., Fu, Y., Sokale, J. R., Leuchter, R. C., and Lagasse, L. D. 
Adenocarcinoma of the uterine cervix: Histologic variables associated with lymph node metastases and survival, Obstet. Gynecol. 65, 46 (1985).

9. Lagasse, L. D., Smith, M. L., Moore, J. G., Morton, D. G., Jacobs, M., Johnson, G. H., and Watring, W. G. The effect of radiation therapy on pelvic lymph node involvement in Stage I carcinoma of the cervix, Amer. J. Obstet. Gynecol. 119, 328 (1974).

10. McDonald, T. W., Morley, G. W., Choo, Y. C., Shields, J. J., Cordoba, R. B., and Naylor, B. Fine needle aspiration of para-aortic and pelvic lymph nodes showing lymphangiographic abnormalities, Obstet. Gynecol. 61, 383 (1983).

11. Bandy, L. C., Clarke-Pearson, D. L., Silverman, P. M., and Creasman, W. T. Computed tomography in evaluation of extrapelvic lymphadenopathy in carcinoma of the cervix, Obstet. Gynecol. 65, 73 (1985). 